\title{
Smart Distance Lab's art fair, experimental data on social distancing during the COVID-19 pandemic
}

\author{
Charlotte C. Tanis ${ }^{1, *}$, Nina M. Leach ${ }^{1}$, Sandra J. Geiger ${ }^{1}$, Floor H. Nauta ${ }^{1}$, Fabian \\ Dablander $^{1}$, Frenk van Harreveld ${ }^{1,2}$, Sanne de Wit ${ }^{1}$, Gerard Kanters ${ }^{3}$, Jop Knoppers ${ }^{3}$, \\ Diederik A. W. Markus ${ }^{3}$, Rick R. M. Bouten ${ }^{4}$, Quinten H. Oostvogel ${ }^{4}$, Meier J. Boersma ${ }^{5}$, \\ Maya V. van der Steenhoven ${ }^{5}$, Denny Borsboom ${ }^{1}$, and Tessa F. Blanken ${ }^{1, *}$ \\ ${ }^{1}$ University of Amsterdam, Department of Psychology, Amsterdam, 1018 WS, the Netherlands \\ ${ }^{2}$ National Institute for Public Health and the Environment (RIVM), Bilthoven, $3721 \mathrm{MA}$, the Netherlands \\ ${ }^{3}$ Centillien B.V., Mierlo, 5731 SG, the Netherlands \\ ${ }^{4}$ Focus Technologies B.V., Eindhoven, 5657 EW, the Netherlands \\ ${ }^{5}$ Smart Distance Lab, Leiderdorp, 2353 NM, the Netherlands \\ *corresponding authors: Charlotte C. Tanis (c.c.tanis@uva.nl) and Tessa F. Blanken (t.f.blanken@uva.nl)
}

13 ABSTRACT

In the absence of a vaccine, social distancing behaviour is pivotal to mitigate COVID-19 virus spread. In this large-scale behavioural experiment, we gathered data during Smart Distance Lab: The Art Fair $(n=839)$ between August 28 and 30, 2020 in Amsterdam, the Netherlands. We varied walking directions (bidirectional, unidirectional, and no directions) and supplementary interventions (face mask and buzzer to alert visitors of 1.5 metres distance). We captured visitors' movements using cameras, registered their contacts (defined as within 1.5 metres) using wearable sensors, and assessed their attitudes toward COVID-19 as well as their experience during the event using questionnaires. We also registered environmental measures (e.g., humidity). In this paper, we describe this unprecedented, multi-modal experimental data set on social distancing, including psychological, behavioural, and environmental measures. The data set is available on figshare and in a MySQL database. It can be used to gain insight into (attitudes toward) behavioural interventions promoting social distancing, to calibrate pedestrian models, and to inform new studies on behavioural interventions.

\section{Background \& Summary}

The COVID-19 pandemic severely affected cultural life around the globe, including theatres, exhibitions, museums, and music events $^{1}$. Many countries, including the Netherlands, shut down cultural venues and generally recommended individuals to maintain a distance of 1.5 metres from one another to reduce virus spread ${ }^{2}$. As the pandemic persisted, a key question became whether behavioural interventions could promote social distancing without bringing society to a standstill. Together with Smart Distance Lab, we conducted a field experiment during an art fair in Amsterdam, the Netherlands, to investigate how social distancing can be promoted effectively during large-scale events. Specifically, we implemented several behavioural interventions and assessed their effectiveness in promoting social distancing behaviour. In doing so, we aim to provide insight into how events can be organised safely during a pandemic.

The art fair was organised between August 28 - 30, 2020 and visited by 839 individuals. The study took place between the first and second COVID-19 wave, when about 500 new COVID-19 cases were registered in the Netherlands each day ${ }^{3}$. We implemented a combination of several interventions, including walking directions (bidirectional, unidirectional, no directions) and supplementary interventions (face mask, buzzer via wearable social distancing sensors, none). Before visiting the art fair, visitors completed a questionnaire, which included questions on factors related to adopting social distancing (e.g., perceived risk, norms, and knowledge), see Figure 1 for a schematic overview. During the visit, we collected distancing data via wearable Social Distancing Sensors (SDSs), movement data via cameras, and indoor environment data such as humidity and temperature. After the visit, we administered an exit questionnaire focusing on experiences with keeping distance during the art fair (e.g., perceived difficulty, adherence, and automaticity). These different data collection modes resulted in a unique data set combining psychological, behavioural, and environmental measures of a large-scale event organised during the COVID-19 pandemic.

The reported data set can, for example, be valuable to provide insight into attitudes and behaviours during the COVID-19 pandemic, to help calibrate pedestrian models, to validate social distancing measurements, and to design subsequent studies investigating behavioural interventions. We initially used these data to investigate how behavioural interventions influence social distancing behaviour, employing Behavioural Contact Networks ${ }^{4}$ (BECONs) that encode which individuals came within 1.5 
metres of each other. We subsequently compared the networks across conditions to assess the effectiveness of the interventions in terms of social distancing ${ }^{5,6}$.

\section{Methods}

\section{Design}

At the art fair, a selection of top graduates of Dutch art academies who finalised their studies in 2019 and 2020 displayed their work in the Kromhouthal in Amsterdam, see Figure 2. Different time slots during the three-day event allowed for implementing different conditions, as shown in Table 1. The original set-up was a fully crossed design of walking directions (bidirectional, unidirectional, no directions) and supplementary interventions (face mask, buzzer, none). However, we had to adapt this set-up due to unforeseen circumstances. As a result, we could only implement experimental conditions during eight of the 11 available time slots. We also needed to repeat the buzzer condition on day 3 because the buzzer settings differed across conditions (see setting specification in Table 1).

In the unidirectional and bidirectional conditions, walking directions were indicated through arrows displayed on the floor. On day 1, arrows were pasted in two directions forming two lanes that guided visitors to walk either clock- or anticlockwise (bidirectional walking condition). On day 2, arrows pointed only in one direction (unidirectional walking condition), while there were no directions on day 3. Within each of these conditions (i.e., during different time slots within each day), we implemented a set of supplementary interventions. For the face mask condition, we handed out face masks to visitors. At the beginning of the buzzer conditions, we operated the SDS such that the sensor would buzz when visitors came within 1.5 metres from one another.

\section{Sample}

In total, 997 tickets were sold for the art fair. During three days, 839 people entered the fair, of which 639 (76.2\%) wore an SDS. As shown in Table 1, we measured eight out of 11 time slots. In time slot 5 and 8, 74 and 132 visitors, respectively, already entered the art fair. Some of these visitors had already left before the SDSs were handed out. Thus, the percentage of visitors who wore an SDS during the experimental conditions was higher than the reported 76.2\%. We gathered demographic information $(n=857)$ when people obtained a ticket. The average age of this group was $45.2(S D=16.0, M i n=12, \operatorname{Max}=82)$, $54.1 \%$ were female, and the majority $(83.9 \%)$ completed higher education. Figure 1 provides a schematic overview of the different visitor streams.

\section{Materials Questionnaires}

Participants completed two questionnaires: a pre-questionnaire before entering the art fair and a post-questionnaire after attending the event. The pre-questionnaire recorded participants' demographics (i.e., age, gender, and educational level), email addresses, and whether they had previously been infected with the coronavirus. It included seven items about a potential coronavirus infection: the likelihood of getting infected ( 0 very unlikely to 100 very likely), the severity ( 1 not serious at all to 7 very serious), the perceived health risk for family and friends, and (separately) for themselves (1 extremely small to 7 extremely large), as well as worries about getting infected, infecting others, and an overloaded healthcare system (1 no worries at all to 7 a lot of worries). The pre-questionnaire also contained 16 items about their attitudes and self-reported behaviours regarding the behavioural guidelines, the perceived social norm, and automaticity of keeping distance (four items of the Self-Reported Behavioral Automaticity Index ${ }^{7} ; 1$ completely disagree to 7 completely agree), as well as how participants experience the social distancing rule (1 to 7, not sensible-sensible, useless-useful, not enjoyable-enjoyable, unfair-fair, unacceptable-acceptable, difficult-easy). Finally, participants were asked about their general health (1 very bad to 7 very good), how they feel about face masks as protection against the coronavirus, and whether it is less important to keep distance when wearing a face mask (1 completely disagree to 7 completely agree). Online-only Table 1 shows both the original Dutch and translated English questions and response options of the pre-questionnaire.

The post-questionnaire recorded participants' email addresses to link the two questionnaires. It included 13 items about participants' social distancing behaviour during the event: whether they tried to maintain distance during the event (1 completely disagree to 7 completely agree), the experienced difficulty of maintaining distance and determining when someone is within 1.5 metres distance ( 1 difficult to 7 easy), their adherence to the 1.5 metre guideline ( 1 not at all to 7 constantly), as well as automaticity of distancing, and whether they felt they were constantly reminded of maintaining distance (1 completely disagree to 7 completely agree). Next, participants were asked to what extent they felt protected against the coronavirus, their stress level during the event, the extent to which they experienced freedom to behave as they wished, to what extent they felt obligated to behave in a certain way (two items), trust in their ability to maintain distance, and pleasure during the event (1 completely disagree to 7 completely agree). Lastly, they were asked how many units of alcohol they consumed during the art fair and 
whether they wore a face mask during their visit. Online-only Table 1 shows both the original Dutch and translated English questions and response options of the post-questionnaire.

\section{Social Distancing Sensor}

SDSs are wearable electronic devices that use ultra-wideband (UWB) technology to detect the presence of other sensors, and measure distance with an accuracy up to ten centimetres. The SDSs in this study were designed by Focus Technologies B.V. (https://www.findfocus.nl) together with Sentech B.V. (https://www.sentech.nl). Data collection required four types of devices: the SDSs, an access point, a laptop connected to the access point running a control application, and multiple base stations. Each sensor had a unique tag ID and locally stored counts of how often other sensors had been within a pre-specified range, i.e., 1.5 metres. The access point located near the entrance of the gallery area collected these counts when an SDS was within 30 metres (line of sight) and sent the data to a central database. A time stamp was only recorded when the data moved from locally stored on the SDS to the central database and, therefore, does not refer to the time of contact itself. Contacts that occurred near the entrance may have been sent to the database immediately, whereas the majority of SDSs were only close enough to the access point when a data sweep was performed. In addition, the access point updated the settings of the SDSs in case they had been changed via the application. An SDS was (de)activated by placing a sensor on a base station. In our set-up, up to four SDSs could be linked by simultaneously placing them on different base stations to avoid registering contacts between members of the same household. As soon as an SDS was deactivated, any other SDSs that were linked to it were disconnected.

The SDSs were set to register a contact when another SDS was within 1.5 metres. When two SDSs were in contact, they gave at least one of three types of feedback: a flashing light, a buzzing sensation, or a beeping sound. This feedback could either occur immediately or after two seconds of contact. Except in the buzzer conditions, we set the feedback to a flashing light and placed the SDS in a small black bag to avoid visitors being able to see whether they were within 1.5 metres of others. This way, data could be gathered without the flashing light influencing the behaviour of visitors. Visitors wore the SDS on a key cord (lanyard) around their neck. In addition to the SDSs worn by visitors, we positioned nine "location" SDSs at a fixed location inside the fair, see the red dots in Figure 2. These sensors were only activated outside of the buzzer conditions to prevent inappropriate feedback to visitors when standing close to a location SDS.

\section{Cameras}

We mounted six optical cameras on trusses at a height of 12 metres. The cameras were configured to record with a resolution of $640 \times 480$ pixels to ensure that visitors of the art fair could not be recognised from the recorded images. Each camera lens had a field of view of $98^{\circ} \times 55^{\circ}$ resulting in a maximum ground coverage of $27.6 \times 12.5$ metres per camera. Three cameras were mounted above the entrance and covered the entire entrance area. The other three cameras were located above the gallery, covering stands 1 to 17 . One of these cameras, covering stands 15 to 17 and the entrance to the toilet, was mounted at an angle of $10^{\circ} \pm 2^{\circ}$ to prevent walls from blocking the camera view. The cameras were configured to record at 10 frames per second (FPS) to enable real-time data processing.

\section{Indoor Environment Measures}

During the event, the temperature, humidity, and light intensity were continuously monitored using the internal sensors of an Ubibot $\mathrm{WS}^{8}{ }^{8}$. The internal temperature sensor had a precision of $\pm 0.3^{\circ} \mathrm{C}$ and a range of $-20^{\circ} \mathrm{C}$ to $60^{\circ} \mathrm{C}$. The internal humidity sensor had a precision of $\pm 3 \mathrm{RH}$ within the range of $10 \%$ to $90 \%$ relative humidity. The light sensor had a precision of $\pm 2 \%$ in the range of 0.01 to $83 \mathrm{~K}$ lux. The indoor environment conditions were sampled every 5 minutes at an approximate height of 2.5 metres from ground level. The red letter "E" in Figure 2 shows the location where the environmental measures took place.

\section{Procedures}

Participants were recruited by promoting the art fair on social media. Before buying a ticket, participants were asked to provide informed consent and complete the online pre-questionnaire, see Figure 1. Two links to the pre-questionnaire existed: one gave access to a free ticket and the other to a ticket that cost 5 euros. The content of both online pre-questionnaires was identical and we merged their responses. Each ticket was associated with a time slot during which visitors could enter the fair. At the fair, tickets were scanned, visitors walked by the cloakroom, and arrived at the research desk. At the research desk, we checked if visitors had filled in the pre-questionnaire using their email address. If not, e.g., because someone else bought their ticket, we only asked visitors to provide informed consent to participate in the study. To avoid congestion near the entrance, we did not ask these visitors to complete the pre-questionnaire on site. Next, an SDS attached to a key cord was handed out, and the visitor's email address was registered to link the SDS and questionnaire data. If visitors came in groups, the linked tag IDs were registered. After registration, visitors activated their SDS at the activation desk, where they also received a small black bag to cover their sensor. We asked participants to activate their SDS themselves to avoid touching the disinfected materials. In the face mask condition, visitors were provided with a face mask and were asked to wear it until the end of the condition.

Once inside the fair, visitors could stay as long as desired. The maximum capacity inside was 150 visitors, which was never exceeded. Before exiting, visitors handed back the SDS, key cord, and bag at the back of the research desk, where their tag ID 
was once again registered. Finally, visitors were asked to scan a QR-code and complete the post-questionnaire. All materials were then disinfected, and the SDS charged before handed out again.

Between conditions, we performed two "data sweeps" by walking through the hall with the laptop and access point. The first collected all data from the sensors in the previous condition. The second activated the settings of the SDSs for the following condition. In addition, after the face mask condition, we informed visitors that they could take off their face mask; and when switching to or from a buzzer condition, we took in or handed out the black bags around the sensors. The camera and indoor environment measurements were performed during the entire art fair and did not require any interaction with visitors.

\section{Ethical Issues}

The University of Amsterdam collected the questionnaire and sensor data. The ethics review board of the University of Amsterdam (2020-CP-12488) approved data collection, and all participants provided informed consent before participating. The camera and indoor environment data were collected and processed by Centillien B.V., a Dutch company specialised in artificial intelligence and image recognition (https://centillien.com). Visitors were informed that the venue was filmed when they obtained a ticket. All personal identifiable information used to link the questionnaire and sensor data has been destroyed. The Ministry of Economic Affairs and Climate Policy (Ministerie van Economische Zaken en Klimaat, EZK) and Mondriaan fund invested in the production costs of the Art Fair and Smart Distance Lab organization.

\section{Data Records}

The data set is available in comma-separated value (CSV) files on figshare $^{9}$, and a relational (MySQL) database hosted by SURF SARA $^{10}$. On figshare, the data are grouped based on the data source (i.e., questionnaire, sensor, camera, and environment). The database can be accessed via mysqlonubuntud.smartdist-uva.src.surf-hosted.nl/phpmyadmin, using the read-only account with username "sdl_guest" and password "dmebozY07tRfigfm", or via any compatible analysis software or app. An example script to connect to the database via R can be found here: https://osf.io/2ag9z/. Table 2 provides an overview and short description of all data tables. Tables 3 - 7 describe each variable for each type of data table.

\section{Technical Validation}

When processing the data, both data checks and cleaning were conducted. The two pre-questionnaires were merged by email address. If participants completed a questionnaire multiple times, only the first completed questionnaire with a unique combination of email address, age, and gender was kept. A questionnaire ID (QID) was automatically assigned to participants who completed a questionnaire. Email addresses were replaced by their corresponding QID in both the questionnaire and sensor data to anonymise the data. We generated unique QIDs for participants in the sensor data who had not completed a questionnaire. We used QIDs instead of tag IDs to link the data sets since the SDSs were handed out multiple times a day to different visitors (see Figure 1).

Contacts are stored twice in the sensor data, because a contact involves two SDSs. Both kept a record with their ID as reporting tag ID and the other as opposing tag ID. However, the sensor data also contained exact duplicates in the database, i.e., the same number of contacts with the same reporting and same opposing tag ID at the same time. In these cases, we only kept one of the records. We also removed a record if an SDS tag ID could not be linked to a QID of a person that was present at that time. These records could occur when an SDS was activated but not handed out, e.g., when removed from the charger and the SDS automatically activated. We added records to the sensor data when an SDS made zero contacts since the data should also include people without any contacts. These visitors were identified using the registration of tag IDs at the beginning and end of a visit. Finally, for each condition, we only kept the sensor data between the start and end data sweep of that condition.

The camera data were processed and described in multiple layers, see Figure 3. The layers follow a hierarchical structure such that each layer serves as an input to the next layer and increases the abstraction of the data ${ }^{11}$. Layer 1 contains the raw video footage captured during the art fair and is available upon request. Layer 2 provides the first level of abstraction from the raw video footage. Computer vision was used to obtain pixel coordinates of visitors. This abstraction was realised by using a sophisticated implementation of a blob detector, which included a K-nearest neighbour (KNN) background subtractor, morph dilation to reduce noise, and chain approximation. In this layer, the data of multiple cameras were merged into one single data set, and we removed data points where the camera views overlap. Each pixel has a width and height of 5.5 centimetres. The pixel coordinates obtained in both layer 2 and 3 can be converted to physical coordinates. The left upper corner of the map corresponds to the point where $x=0$ and $y=0$. Layer 3 adds a second level of abstraction by including time information to allow tracking of visitors over time. A centroid tracking algorithm in combination with filtering was used to provide data points where time gaps were reconstructed, and noise in the spatial-time domain was removed.

The indoor environment measures were taken at a height of 2.5 metres to prevent visitors from accessing the device. 


\section{Usage Notes}

For each table we provide a code book. We recommend reading the code book before accessing the data tables. To connect to the MySQL database, you might need the following information:

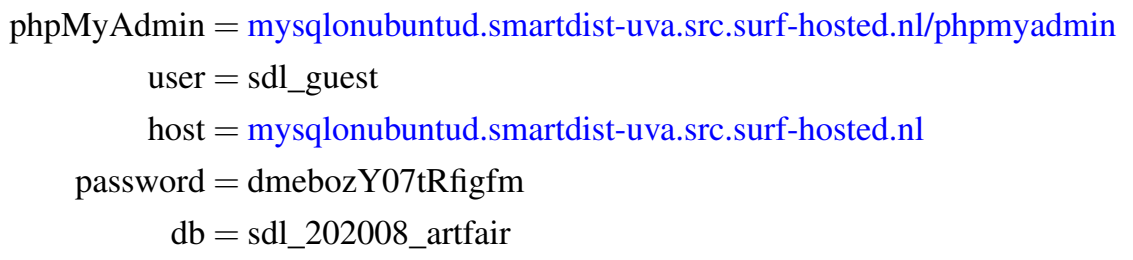

\section{Code Availability}

All code related to this data set can be found in the Smart Distance Lab OSF project ${ }^{12}$.

\section{References}

1. van Leeuwen, M., Klerks, Y., Bargeman, B., Heslinga, J. \& Bastiaansen, M. Leisure will not be locked down - insights on leisure and COVID-19 from the Netherlands. World Leis. J. 62, 339-343, https://doi.org/10.1080/16078055.2020.1825255 (2020).

2. Rijksoverheid. Corona en regels voor afstand houden. https://www.rijksoverheid.nl/onderwerpen/coronavirus-covid-19/ openbaar-en-dagelijks-leven/afstand-houden (2020).

3. Dong, E., Du, H. \& Gardner, L. An interactive web-based dashboard to track COVID-19 in real time. The Lancet Infect. Dis. 20, 533-534, https://doi.org/10.1016/S1473-3099(20)30120-1 (2020).

4. Borsboom, D. et al. The lighting of the BECONs: A behavioral data science approach to tracking interventions in COVID-19 research. Preprint at https://psyarxiv.com/53ey9 (2020).

5. Smart Distance Lab. Bevindingen. https://smartdistancelab.nl/bevindingen-2/ (2020).

6. Blanken, T. F. et al. The Smart Distance Lab: A new methodology for assessing social distancing interventions. Preprint at https://osf.io/mjg2f (2020).

7. Gardner, B., Abraham, C., Lally, P. \& de Bruijn, G. J. Towards parsimony in habit measurement: Testing the convergent and predictive validity of an automaticity subscale of the Self-Report Habit Index. Int. J. Behav. Nutr. Phys. Activity 9 , 102, https://doi.org/10.1186/1479-5868-9-102 (2012).

8. UbiBot. UbiBot WS1 User Guide. https://www.ubibot.com/wp-content/uploads/dlm\{_\}uploads/2020/12/ WS1-User-Guide-English.pdf (2020).

9. Tanis, C. C. et al. Data record of: Smart Distance Lab Art Fair - An experimental dataset on social distancing during the COVID-19 pandemic. figshare https://doi.org/10.6084/m9.figshare.c.5276678.v2 (2021).

10. Tanis, C. C. et al. Smart Distance Lab: Relational MySQL Database. http://sdl.smartdist-uva.surf-hosted.nl/ (2021).

11. Knoppers, J., Markus, D. A. W. \& Kanters, G. Fever detection, distance measurement, headcount and group formation analysis using computer vision software Intra to detect SARS-CoV-2 regulation violations. https://centillien.com/wp-content/ uploads/2020/11/Whitepaper-Covid-19-measurements.pdf (2020).

12. Blanken, T. F., Tanis, C. C., Borsboom, D. \& van Harreveld, F. Smart Distance Lab. OSF https://doi.org/10.17605/osf.io/ u9avy (2021).

\section{Acknowledgements}

We would like to thank all volunteers that helped collecting the data: Nicolette Aschermann, Elenika Schalm Boiko, Trijntje van Doorn, Nadza Dzinalija, Melle van der Linde, Henk Nieweg, and Marieke Wagenaar; SVP Sfeerbeheer and de Kromhouthal for organising and hosting the art fair; and Matthijs Immink (https://matthijsimmink.com) for allowing us to include his photo of the art fair.

The research project was supported by the Ministry of Economic Affairs and Climate Policy and Mondriaan fund C.C.T., F.D. and T.F.B are supported by NWO Fast Track grant 440.20 .032 
C.C.T. and T.F.B. are supported by an Innovation Exchange Amsterdam UvA Proof of Concept Fund

F.D. is supported by ZonMw project 10430022010001

\section{Author contributions statement}

C.C.T. developed study design and behavioural interventions; gathered data; managed and coordinated production of data set; wrote manuscript

N.M.L. gathered data; set up database; wrote manuscript

S.J.G. gathered data; wrote manuscript

F.H.N. gathered and processed SDS and questionnaire data

F.D. checked the processing of SDS and questionnaire data

F.vH. developed study design, behavioural interventions, and questionnaire content

S.dW. developed study design, behavioural interventions, and questionnaire content

G.K. operated as liaison between Centillien, UvA, and EZK; realised camera and environmental hardware

J.P. gathered and processed camera and environmental data

D.A.W.M. gathered and processed camera and environmental data

R.R.M.B. provided the SDSs

Q.H.O. provided the SDSs

M.J.B. conceived the Smart Distance Lab initiative

M.V.vdS. conceived the Smart Distance Lab initiative

D.B. developed study design and behavioural interventions; gathered data

T.F.B. developed study design and behavioural interventions; gathered data; managed and coordinated data collection and production of data set; wrote manuscript

All authors commented on the manuscript, and reviewed and approved the final manuscript.

\section{Competing interests}

G.K., J.P. and D.A.W.M. are employed by Centillien B.V.

R.R.M.B. and Q.H.O. are employed by Focus Technologies B.V.

\section{Figures \& Tables}




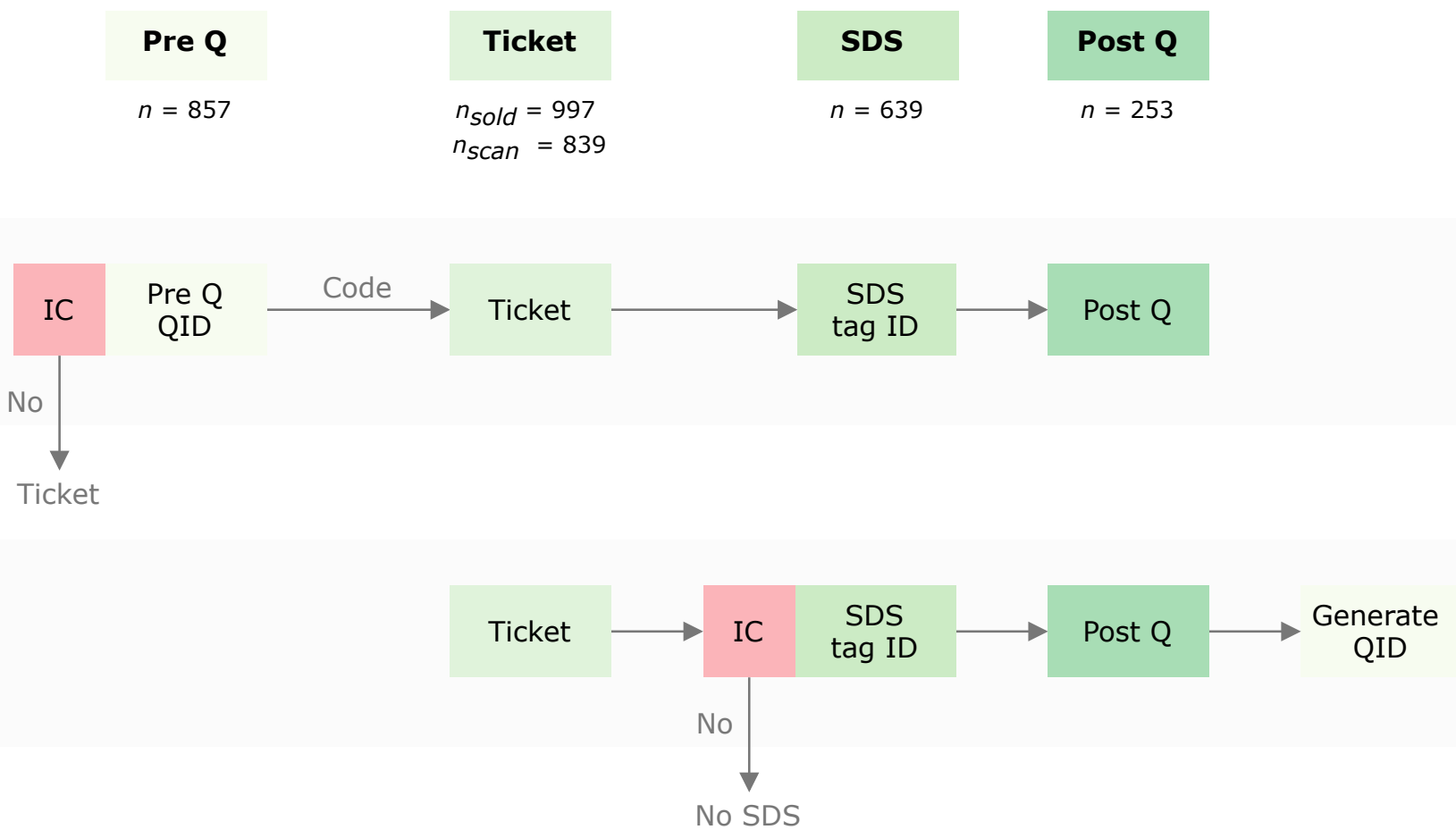

Figure 1. Overview of data collection. The top stream represents visitors who purchased their own ticket. After providing informed consent (IC), visitors completed a pre-questionnaire (Pre Q) which gave access to a code for a ticket. If participants declined informed consent, they received the code immediately. At the art fair, visitors received an SDS before entering and were asked to complete a post-questionnaire (Post Q) when exiting. Visitors in the bottom stream did not complete the pre-questionnaire and were asked to provide informed consent before receiving an SDS. We used the questionnaire IDs (QID) as a unique identifier for participants. For visitors in the bottom stream, we generated QIDs after collecting the data. Camera and indoor environment data were collected throughout the art fair. 


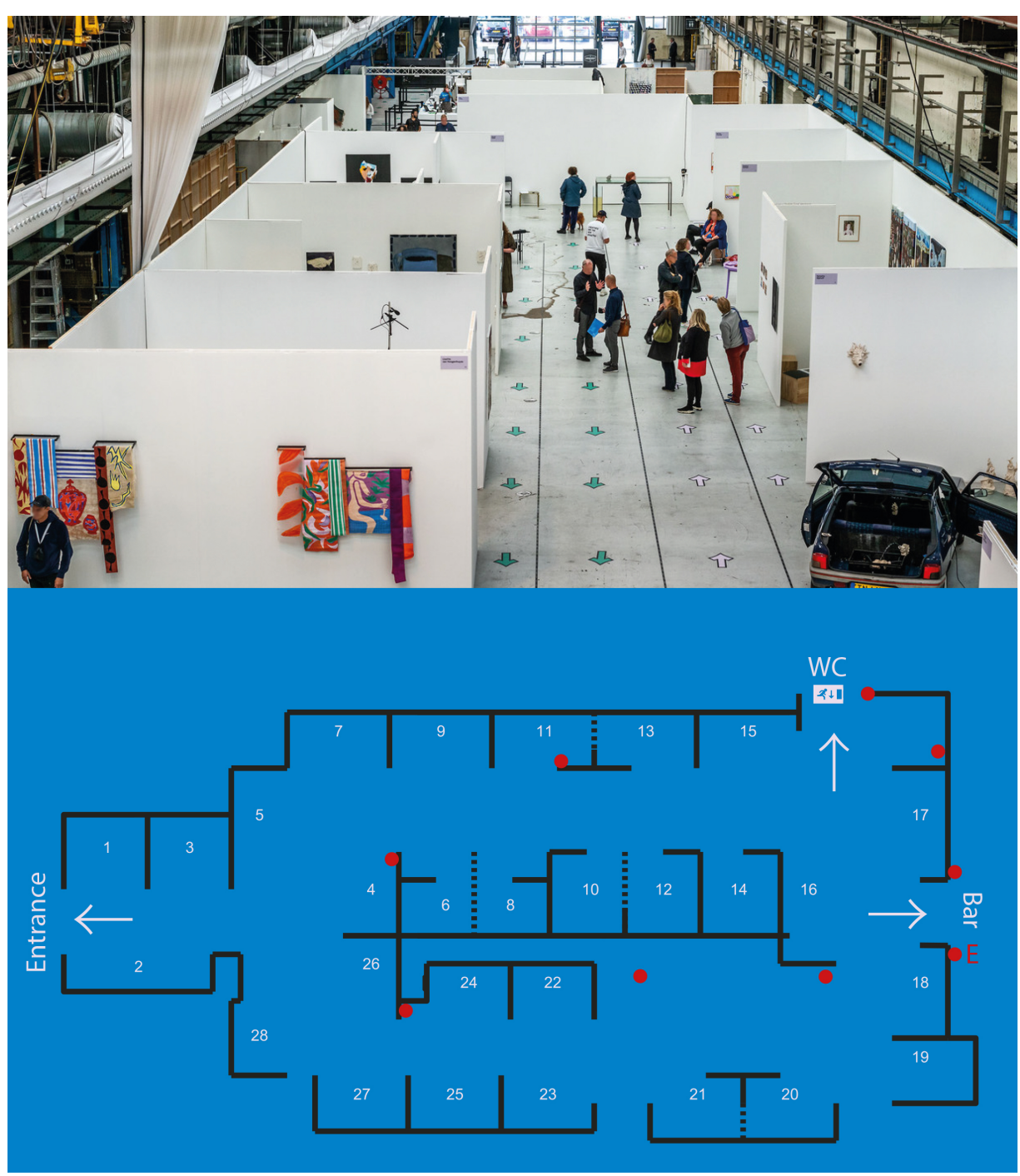

Figure 2. Layout of the art fair. The total area used for the event was divided into three main sections: entrance $\left(500 \mathrm{~m}^{2}\right)$, gallery $\left(1,080 \mathrm{~m}^{2}\right)$, and bar $\left(1,338 \mathrm{~m}^{2}\right)$. Visitors entered on the left side after passing the cloakroom and research desk. The picture was taken on the first day when walking directions were bidirectional. The layout below shows the gallery with the artists' stands $(1-28)$. Stands were on average $16.68 \mathrm{~m}^{2}\left(\operatorname{Min}=13.5 \mathrm{~m}^{2}, \operatorname{Max}=19.5 \mathrm{~m}^{2}\right)$. Due to the layout of the stands, the accessible area in the gallery was smaller than the total floor area. The red dots indicate the fixed places of the nine location badges. The indoor environment was measured at the red letter "E". 


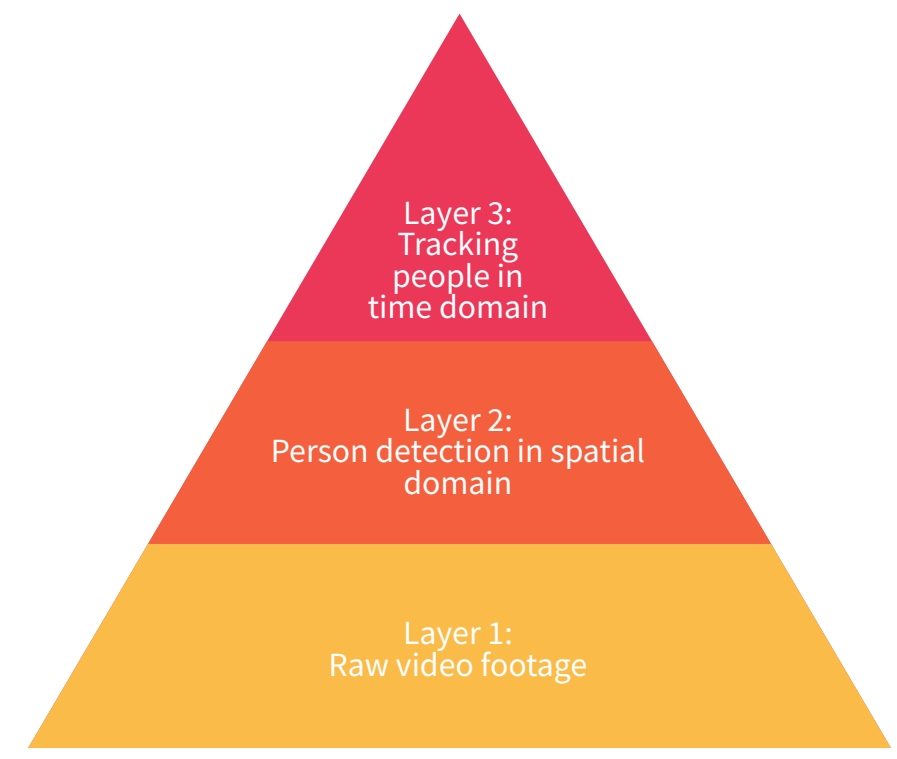

Figure 3. The layered structure of the camera data can be represented as a pyramid. Each layer depends on the previous layer and increases the abstraction of the data.

\begin{tabular}{|c|c|c|c|c|c|c|c|c|}
\hline $\begin{array}{l}\text { Time } \\
\text { slot }\end{array}$ & Day & Start & End & $\begin{array}{l}\text { Walking } \\
\text { direction }\end{array}$ & $\begin{array}{l}\text { Supplementary } \\
\text { intervention }\end{array}$ & SDS setting & $\mathrm{N}$ & $\begin{array}{l}\text { Duration } \\
\text { (min) }\end{array}$ \\
\hline 1 & 28-Aug & 08:00 & $11: 30$ & Bidirectional & & No SDS & & \\
\hline 2 & 28-Aug & $13: 30$ & $15: 30$ & Bidirectional & Facemask & No feedback ${ }^{a}$ & 130 & 120 \\
\hline 3 & 28-Aug & $15: 30$ & $17: 30$ & Bidirectional & None & No feedback ${ }^{\mathrm{a}}$ & 137 & 120 \\
\hline 4 & 28-Aug & $17: 30$ & $19: 30$ & Bidirectional & Buzzer & Buzzer after $2 \mathrm{sec}$ & 122 & 120 \\
\hline 5 & 29-Aug & 11:00 & $13: 30$ & Unidirectional & & No SDS & & \\
\hline 6 & 29-Aug & $13: 30$ & $15: 30$ & Unidirectional & None & No feedback ${ }^{\mathrm{a}}$ & 147 & 120 \\
\hline 7 & 29-Aug & $16: 00$ & $18: 00$ & Unidirectional & Buzzer & $\begin{array}{l}\text { Buzzer immediately, } \\
\text { stops after } 2 \mathrm{sec}\end{array}$ & 137 & 120 \\
\hline 8 & 30-Aug & 11:00 & $13: 30$ & No direction & & No SDS & & \\
\hline 9 & 30-Aug & $13: 30$ & $15: 30$ & No direction & Buzzer & $\begin{array}{l}\text { Buzzer immediately, } \\
\text { stops after } 2 \mathrm{sec}\end{array}$ & 123 & 120 \\
\hline 10 & 30-Aug & $15: 30$ & $16: 30$ & No direction & Buzzer & $\begin{array}{l}\text { Buzzer immediately, } \\
\text { persists after } 2 \mathrm{sec}\end{array}$ & 146 & 60 \\
\hline 11 & 30-Aug & $17: 00$ & 18:00 & No direction & None & No feedback ${ }^{\mathrm{a}}$ & 102 & 60 \\
\hline
\end{tabular}

Table 1. Descriptives per condition. We implemented experimental conditions in eight time slots $(2,3,4,6,7,9$, 10, and 11). Some of these time slots contained only walking directions (Supplementary intervention $=$ None), while others contained both walking directions and a supplementary intervention. SDSs were not handed out during the remaining time slots $(1,5$, and 8$)$. Six experimental conditions lasted two hours, while two lasted one hour. Except in the buzzer conditions, the SDS was covered in a black bag so the flashing light was invisible. Note that the sum of the number of visitors across conditions differs from the reported $n=639$ visitors who wore an SDS, because some people stayed inside the art fair during multiple conditions.

${ }^{a}$ The SDS requires to always select at least one form of feedback (light, buzzer, sound). In conditions during which the SDS should not provide feedback (face mask, no supplementary intervention), we set the settings to light, and provided black bags to cover the SDS, such that no feedback was received. 


\begin{tabular}{|c|c|c|}
\hline Group & Table & Description \\
\hline & conditions & Overview conditions during art fair \\
\hline camera & camera_codebook & Codebook \\
\hline camera & camera_layer2_all & $\begin{array}{l}\text { Unfiltered point detections of people } \\
\text { from the raw footage }\end{array}$ \\
\hline camera & camera_layer3_20200828_02_bidirectional_facemask_120 & $\begin{array}{l}\text { Layer } 3 \text { data of time slot } 2 \text { excluding } \\
\text { entrance area, } \mathrm{x} \text { between } 0 \text { and } 845\end{array}$ \\
\hline camera & camera_layer3_20200828_03_bidirectional_nointervention_120 & $\begin{array}{l}\text { Layer } 3 \text { data of time slot } 3 \text { excluding } \\
\text { entrance area, } x \text { between } 0 \text { and } 845\end{array}$ \\
\hline camera & camera_layer3_20200828_04_bidirectional_buzzer_120 & $\begin{array}{l}\text { Layer } 3 \text { data of time slot } 4 \text { excluding } \\
\text { entrance area, } \mathrm{x} \text { between } 0 \text { and } 845\end{array}$ \\
\hline camera & camera_layer3_20200829_06_unidirectional_nointervention_120 & $\begin{array}{l}\text { Layer } 3 \text { data of time slot } 6 \text { excluding } \\
\text { entrance area, } x \text { between } 0 \text { and } 845\end{array}$ \\
\hline camera & camera_layer3_20200829_07_unidirectional_buzzer_120 & $\begin{array}{l}\text { Layer } 3 \text { data of time slot } 7 \text { excluding } \\
\text { entrance area, } x \text { between } 0 \text { and } 845\end{array}$ \\
\hline camera & camera_layer3_20200830_09_nodirection_buzzer_120 & $\begin{array}{l}\text { Layer } 3 \text { data of time slot } 9 \text { excluding } \\
\text { entrance area, } \mathrm{x} \text { between } 0 \text { and } 845\end{array}$ \\
\hline camera & camera_layer3_20200830_10_nodirection_buzzer_60 & $\begin{array}{l}\text { Layer } 3 \text { data of time slot } 10 \text { excluding } \\
\text { entrance area, } \mathrm{x} \text { between } 0 \text { and } 845\end{array}$ \\
\hline camera & camera_layer3_20200830_11_nodirection_nointervention_60 & $\begin{array}{l}\text { Layer } 3 \text { data of time slot } 11 \text { excluding } \\
\text { entrance area, } \mathrm{x} \text { between } 0 \text { and } 845\end{array}$ \\
\hline camera & camera_layer3_all & $\begin{array}{l}\text { Filtered point detections of labeled } \\
\text { individuals }\end{array}$ \\
\hline camera & camera_map_all & Coordinates of walls in the art fair \\
\hline camera & camera_map_codebook & Codebook \\
\hline environment & environment_all & $\begin{array}{l}\text { Temperature, humidity and light mea- } \\
\text { sures }\end{array}$ \\
\hline environment & environment_codebook & Codebook \\
\hline questionnaire & questionnaire_all & $\begin{array}{l}\text { Responses to pre- and post- } \\
\text { questionnaire }\end{array}$ \\
\hline questionnaire & questionnaire_codebook & Codebook \\
\hline sensor & sensor_all & SDS data of all conditions \\
\hline sensor & sensor_codebook & Codebook \\
\hline sensor & sensor_degree_all & $\begin{array}{l}\text { Number of unique contacts per partic- } \\
\text { ipant }\end{array}$ \\
\hline sensor & sensor_degree_codebook & Codebook \\
\hline
\end{tabular}

Table 2. Overview of data tables. All tables are available as .csv files on figshare ${ }^{9}$, and in a relational (MySQL) database ${ }^{10}$. 


\begin{tabular}{|c|c|c|c|}
\hline Name & Description & Type & Values \\
\hline timestamp & $\begin{array}{l}\text { Timestamp of SDS data collected by access point } \\
\text { in ISO } 8601 \text { format, timezone is CEST }\end{array}$ & String & YYYY-MM-DDTHH:mm:ss \\
\hline day & Event day & Integer & $\begin{array}{l}1=2020-08-28 \\
2=2020-08-29 \\
3=2020-08-30\end{array}$ \\
\hline reporting_tagid & SDS tag id reporting contact & Integer & $146-1176$ \\
\hline reporting_qid & Unique person identifier wearing reporting SDS & Integer & $11-998937$ \\
\hline opposing_tagid & SDS tag id opposing contact & Integer & $146-1176$ \\
\hline opposing_qid & Unique person identifier wearing opposing SDS & Integer & $11-998937$ \\
\hline $\mathrm{n} \_$incidents & Number of contacts & Integer & $0-224$ \\
\hline timeslot & Time slot number & Integer & $2-11$ \\
\hline direction & Walking directions & Integer & $\begin{array}{l}0=\text { no directions } \\
1=\text { unidirectional } \\
2=\text { bidirectional }\end{array}$ \\
\hline pre_q & Completed pre-questionnaire & Integer & $\begin{array}{l}0=\text { not completed } \\
1=\text { completed }\end{array}$ \\
\hline post_q & Completed post-questionnaire & Integer & $\begin{array}{l}0=\text { not completed } \\
1=\text { completed }\end{array}$ \\
\hline linked & SDS linked to household / group members & Integer & $\begin{array}{l}0=\text { not linked } \\
1=\text { at least } 1 \text { linked SDS }\end{array}$ \\
\hline linked_id1 & SDS tag id of first linked SDS & Integer & $146-1176$ \\
\hline linked_id2 & SDS tag id of second linked SDS & Integer & $146-1157$ \\
\hline linked_id3 & $\begin{array}{l}\text { SDS tag id of third linked SDS, } \\
\text { note no groups of } 4\end{array}$ & Integer & NA \\
\hline location_tag & $\begin{array}{l}\text { SDS has fixed location in art fair, } \\
\text { note location tags do not have a QID }\end{array}$ & Integer & $\begin{array}{l}0=\text { SDS worn by visitor } \\
1=\text { fixed location SDS }\end{array}$ \\
\hline
\end{tabular}

Table 3. Description of the sensor data. QID is unique per person, while tag ID refers to the SDS and was handed out to multiple visitors during the art fair. A contact between two SDSs was registered on both sensors. Each sensor sent the data to the access point with their ID as reporting ID, and the other as opposing ID. When visitors from the same household linked their SDSs, their contacts were not registered.

\begin{tabular}{llll}
\hline \multicolumn{1}{c}{ Name } & \multicolumn{1}{c}{ Description } & Type & \multicolumn{1}{c}{ Values } \\
\hline qid & Unique person identifier & Integer & $11-998937$ \\
timeslot & Time slot number & Integer & $2-11$ \\
degree & Number of unique contacts within timeslot & Integer & $0-49$ \\
\hline
\end{tabular}

Table 4. Description of the unique contacts data. For each visitor, the number of unique contacts - within 1.5 metres - per time slot.

\begin{tabular}{|c|c|c|c|}
\hline Name & Description & Type & Values \\
\hline timestamp & $\begin{array}{l}\text { Timestamp of detection in ISO } 8601 \text { microseconds } \\
\text { format, timezone is CEST }\end{array}$ & String & YYYY-MM-DDTHH:mm:ss.ssssss \\
\hline id & Unique person identifier, only in layer 3 & Integer & $0-29986$ \\
\hline $\mathrm{x}$ & $\begin{array}{l}x \text { coordinate in pixels, each pixel has a width and } \\
\text { height of } 5.5 \mathrm{~cm}\end{array}$ & Integer & $-99-1567$ \\
\hline $\mathrm{y}$ & $\begin{array}{l}\text { y coordinate in pixels, each pixel has a width and } \\
\text { height of } 5.5 \mathrm{~cm}\end{array}$ & Integer & $300-740$ \\
\hline
\end{tabular}

Table 5. Description of the camera data. The "id" column is only present in the layer 3 tables, as detections have not been linked to unique visitors yet in layer 2. The full layer 2 and 3 tables contain all unfiltered and filtered detections of visitors respectively. The time slot specific tables of layer 3 only contain detections within the gallery area $(0 \leq \mathrm{x} \leq 845)$. 


\begin{tabular}{llll}
\hline Name & \multicolumn{1}{c}{ Description } & Type & \multicolumn{1}{c}{ Values } \\
\hline wallid & Unique wall identifier & Integer & $1-32$ \\
x1 & x coordinate of left corner of wall & Integer & $59-1240$ \\
y1 & y coordinate of top corner of wall & Integer & $394-626$ \\
x2 & x coordinate of right corner of wall & Integer & $62-1250$ \\
y2 & y coordinate of bottom corner of wall & Integer & $366-687$ \\
\hline
\end{tabular}

Table 6. Description of the map for the camera data. The map provides the coordinates of the 32 walls in the area of the art fair where camera data were collected.

\begin{tabular}{|c|c|c|c|}
\hline Name & Description & Type & Values \\
\hline timestamp & $\begin{array}{l}\text { Date and time of measurement in ISO } 8601 \text { format, } \\
\text { timezone is CEST }\end{array}$ & String & YYYY-MM-DDTHH:mm:ss \\
\hline temperature & Temperature in degrees Celsius & Numeric & $19.7-28.3$ \\
\hline humidity & Relative humidity & Integer & $46-60$ \\
\hline light & Light in lux & Numeric & $0-626.9$ \\
\hline
\end{tabular}

Table 7. Description of the environmental data. Temperature, humidity, and light were measured every five minutes during the entire art fair. 